\title{
Reconstruction du savoir-évaluer sous la contrainte : une analyse du bagage d'expériences non réinvesti dans les écoles montréalaises par des enseignants formés à l'étranger
}

\section{Serigne Ben Moustapha Diédhiou}

Volume 8, numéro 2, 2018

L'école à l'épreuve de la diversité ethnoculturelle de son personnel : regards compréhensifs croisés

URI : https://id.erudit.org/iderudit/1066952ar

DOI : https://doi.org/10.7202/1066952ar

Aller au sommaire du numéro

Éditeur(s)

Alterstice

ISSN

1923-919X (numérique)

Découvrir la revue

Citer cet article

Diédhiou, S. B. M. (2018). Reconstruction du savoir-évaluer sous la contrainte : une analyse du bagage d'expériences non réinvesti dans les écoles montréalaises par des enseignants formés à l'étranger. Alterstice, 8(2), 51-62. https://doi.org/10.7202/1066952ar
Résumé de l'article

Cette contribution prend appui sur les résultats d'une recherche doctorale collaborative menée auprès d'enseignants formés à l'étranger qui s'intègrent dans les écoles montréalaises. Au cours des dernières décennies, les écoles sur ce territoire ont accueilli un nombre important d'entre eux en raison de l'urgence de combler des postes d'enseignement laissés vacants par les départs à la retraite, l'attrition des enseignants en début de carrière et l'ouverture de nouvelles classes pour les besoins de scolarisation de tous les enfants, notamment ceux d'origine immigrante. La recherche sur laquelle repose cet article suggère que ces enseignants socialisés dans d'autres contextes nationaux rencontrent de nombreuses difficultés. Sur le plan de l'évaluation des apprentissages notamment, ils doivent adapter leurs façons de faire. Dans cette perspective, j'ai documenté à l'appui de la conceptualisation de la " définition de la situation " de Thomas (1923), les usages de l'évaluation que 5 enseignants formés à l'étranger ayant participé à cette recherche ont faits en s'intégrant dans les écoles montréalaises. Le présent article cible ceux qui sont contestés par leurs partenaires de travail au point où ils ne peuvent les réinvestir dans ce nouveau contexte. L'analyse de ce bagage non réinvesti expose comment ces enseignants abandonnent en particulier deux manières d'utiliser l'évaluation. La première concerne la mobilisation de l'évaluation comme un pouvoir dont la violence symbolique oblige les élèves à obéir à l'enseignant. La deuxième concerne l'application de l'évaluation dans le sens d'une justice méritocratique qui conduit à ne valoriser que les meilleurs élèves. Comme on le verra, ces processus de reconstruction du savoir-évaluer se négocient au coeur d'expériences interactives sous tensions entre les EFE et certains de leurs partenaires scolaires, révélatrices des conventions qui cadrent la pratique de l'évaluation des apprentissages dans les écoles montréalaises.
Ce document est protégé par la loi sur le droit d'auteur. L’utilisation des services d’Érudit (y compris la reproduction) est assujettie à sa politique d'utilisation que vous pouvez consulter en ligne.

https://apropos.erudit.org/fr/usagers/politique-dutilisation/ 
ARTICLE THÉMATIQUE

\title{
Reconstruction du savoir-évaluer sous la contrainte : une analyse du bagage d'expériences non réinvesti dans les écoles montréalaises par des enseignants formés à l'étranger
}

Serigne Ben Moustapha Diédhiou

\begin{abstract}
Résumé
Cette contribution prend appui sur les résultats d'une recherche doctorale collaborative menée auprès d'enseignants formés à l'étranger qui s'intègrent dans les écoles montréalaises. Au cours des dernières décennies, les écoles sur ce territoire ont accueilli un nombre important d'entre eux en raison de l'urgence de combler des postes d'enseignement laissés vacants par les départs à la retraite, l'attrition des enseignants en début de carrière et l'ouverture de nouvelles classes pour les besoins de scolarisation de tous les enfants, notamment ceux d'origine immigrante. La recherche sur laquelle repose cet article suggère que ces enseignants socialisés dans d'autres contextes nationaux rencontrent de nombreuses difficultés. Sur le plan de l'évaluation des apprentissages notamment, ils doivent adapter leurs façons de faire. Dans cette perspective, j'ai documenté à l'appui de la conceptualisation de la " définition de la situation » de Thomas (1923), les usages de l'évaluation que 5 enseignants formés à l'étranger ayant participé à cette recherche ont faits en s'intégrant dans les écoles montréalaises. Le présent article cible ceux qui sont contestés par leurs partenaires de travail au point où ils ne peuvent les réinvestir dans ce nouveau contexte. L'analyse de ce bagage non réinvesti expose comment ces enseignants abandonnent en particulier deux manières d'utiliser l'évaluation. La première concerne la mobilisation de l'évaluation comme un pouvoir dont la violence symbolique oblige les élèves à obéir à l'enseignant. La deuxième concerne l'application de l'évaluation dans le sens d'une justice méritocratique qui conduit à ne valoriser que les meilleurs élèves. Comme on le verra, ces processus de reconstruction du savoir-évaluer se négocient au cœur d'expériences interactives sous tensions entre les EFE et certains de leurs partenaires scolaires, révélatrices des conventions qui cadrent la pratique de l'évaluation des apprentissages dans les écoles montréalaises.
\end{abstract}

\section{Rattachement de l'auteur \\ ${ }^{1}$ Université du Québec à Montréal, Montréal, Canada}

\section{Correspondance}

diedhiou.serigne_ben_moustapha@uqam.ca

\section{Mots clés}

enseignants formés à l'étranger, bagage d'expériences, définition de la situation, école québécoise, enseignants formés à l'étranger, intégration socioprofessionnelle, justice méritocratique, savoir-évaluer, usage de l'évaluation.

\section{Pour citer cet article}

Diédhiou, S.B.M. (2018). Reconstruction du savoir-évaluer sous la contrainte : une analyse du bagage d'expériences non réinvesti dans les écoles montréalaises par des enseignants formés à l'étranger. Alterstice, 8(2), 51-62. 


\section{Introduction}

Depuis quelques décennies, les écoles québécoises sont le théâtre de l'intégration socioprofessionnelle d'enseignants formés à l'étranger (EFE). Ces enseignants sont présents en nombre important dans le système éducatif québécois (au préscolaire, au primaire et au secondaire) car, entre 1998 et 2009, environ 3000 autorisations d'enseigner leur ont été délivrées, et une majorité aurait débouché sur un recrutement (Niyubahwe, Mukamurera et Jutras, 2014). Les données de Services Canada (2015) reflètent que les EFE constituent près de $8 \%$ du personnel enseignant au niveau préscolaire et primaire. Selon plusieurs observateurs, cet effectif serait appelé à s'accroître dans les années à venir, compte tenu de la pénurie d'enseignants observée au Québec récemment (Caza, 2019; Cloutier, 2018). Cependant, s'il ne fait plus de doute que l'intégration socioprofessionnelle des EFE est d’intérêt au Québec pour résorber le manque d'enseignants relevé dans les écoles (Niyubahwe, 2015), il reste que leurs défis par rapport à l'évaluation semblent constituer un objet majeur de préoccupation.

Cette contribution éclaire la reconstruction du savoir-évaluer de cinq enseignants formés à l'étranger (EFE) ayant participé à une recherche doctorale (Diédhiou, 2018), en mettant l'accent sur le bagage d'expériences qu'ils abandonnent en raison des contraintes que leurs partenaires de travail opposent à son réinvestissement dans les écoles montréalaises. Cet article vient souligner que leur intégration socioprofessionnelle se négocie notamment par des renoncements à certaines finalités de l'évaluation contestées par leurs partenaires de travail. En outre, il montre comment les EFE sont socialisés à la prise en compte du bien-être de l'enfant, soit la norme par excellence au Québec (Morrissette et Demazière, 2018). Ce constat conduit à une discussion conclusive sur le fait que l'importance de cette norme pour les partenaires de travail laisse aux EFE très peu de marge de manœuvre en matière d'évaluation.

\section{Une transition de carrière parsemée d'obstacles}

À la faveur de la politique d’immigration québécoise qui cible spécifiquement les immigrants qualifiés (Gouvernement du Québec, 2018), les écoles québécoises ont attiré un grand nombre d’EFE. Après une évaluation comparative de leurs diplômes par le ministère de l'Éducation, plusieurs d'entre eux s'engagent à suivre la formation d'appoint obligatoire pour obtenir le brevet d'enseignement, soit 5 cours dans une institution universitaire (MEES, 2015). Or, cette formation ne semble pas toujours faciliter leur intégration dans les écoles, où ils rencontrent de nombreuses difficultés, alors que dans le contexte actuel, leur intégration socioprofessionnelle est rattachée à des enjeux très importants (Charara et Morrissette, 2018 ; Morrissette, Demazière, Diédhiou et Segueda, 2018).

En effet, sur le plan social, le Québec est confronté à une forte pénurie d'enseignants (Caza, 2019) qui risque de s'aggraver dans les années à venir, alors que les besoins d'encadrement des élèves deviennent de plus en plus importants (Cloutier, 2018). À titre d'exemple, il faut remarquer que l'accueil des immigrants et plus récemment des réfugiés et des demandeurs d'asile a des conséquences sur l'accroissement des effectifs d'élèves dans les écoles et la nécessité d'ouvrir des classes supplémentaires pour accueillir tous ces nouveaux élèves. Sur ce plan, les EFE constitueraient une ressource importante pour aider les commissions scolaires de Montréal à faire face à la crise. Il faut savoir que même s'ils ne sont pas formés au Québec, ils constituent un personnel professionnel hautement qualifié et donc important pour le développement de la société québécoise (Zietsma, 2010). Pour l'école par exemple, il serait important de pouvoir compter sur l'expertise de ces EFE, sachant que différents auteurs soutiennent qu'ils ont une sensibilité particulière pour les élèves récemment immigrés, servant de modèles inspirants pour ces jeunes et facilitant un plus grand sentiment d'appartenance à leur école (Goodson, Thiessen et Bascia, 1997). Sur le plan de la relation école-famille, les EFE seraient des interprètes culturels importants dans la communication entre les directions et les parents d'élèves d'origine immigrante (Goodson, Thiessen et Bascia, 1997 ; Wang, 2003).

Sur le plan scientifique, la recherche sur la question de l'intégration socioprofessionnelle des EFE est donc brûlante d'actualité, d'autant plus qu'on sait encore peu de choses sur ce phénomène grandissant. Les recherches qui s'intéressent à la transition de carrière des EFE en relation avec un changement de contexte d'enseignement ont 
davantage éclairé les obstacles que ceux-ci rencontrent durant ce processus. En effet, certains auteurs ont montré que les difficultés des EFE commenceraient dès les premiers contacts avec le marché du travail, dès l'accès en fait à ce marché (Mujawamariya, 2000), et se prolongeraient dans la formation en enseignement, durant la période de stage (lorsque cette possibilité existe, comme dans la province voisine l'Ontario) (Jabouin et Duchesne, 2012). D'autres auteurs relèvent que ces difficultés seraient aussi vécues en milieu de travail après l'obtention d'un contrat dans une école (Phillion, 2003). Deters (2006) ainsi que Martineau et Vallerand (2007) identifient l'absence d'expérience professionnelle en contexte québécois comme un dénominateur commun des difficultés d'intégration socioprofessionnelle des EFE. Ces derniers s'intégreraient souvent dans les écoles avec une expérience au travail façonnée par une représentation historiquement hiérarchisée des rôles et des pouvoirs des acteurs de l'école (Duchesne, 2008; Myles, Cheng et Wang, 2006). Pour Niyubahwe (2015; voir aussi Niyubahwe, Mukamurera et Jutras, 2013 et 2014), ces écarts avec le contexte d'accueil seraient le point de départ de chocs culturels, de désenchantements et de pertes de repères au début de leur intégration socioprofessionnelle. Dans la recension des écrits qu'ils ont produite, Morrissette, Diédhiou et Charara (2014) ont dégagé de ces travaux que ceux-ci suivent souvent une perspective psychologisante et individualisante de l'intégration socioprofessionnelle, en mettant l'accent sur l'EFE seul et les obstacles qu'il rencontre. En cela, ils minorent, voire passent sous silence, le rôle joué par les autres membres de leur écologie professionnelle dans cette intégration.

Dans cette même recension, d'autres types de difficultés sont répertoriées, par exemple sur le plan des relations avec les différents partenaires de travail (élèves, parents, direction, collègues, etc.) et sur le plan des pratiques pédagogiques. Sur ce dernier point, la plupart du temps ils connaissent mal le programme en vigueur dans la société d'accueil et ils ne sont en outre pas habitués à l'évaluation des apprentissages, en termes de finalités comme de modalités. Si ce constat a été soulevé dans les récents travaux de Morrissette et Demazière (2018), qui ont relevé la distance entre leurs référents sur ce plan et ceux valorisés au Québec, il n'a pas été davantage examiné. Pourtant, la question de l'évaluation constitue aujourd'hui un sujet soulevant de vives tensions entre les acteurs de l'école (ADMÉÉ, 2014). Certes, des travaux soulignent qu'en s'intégrant dans un nouveau milieu de travail, les pratiques d'évaluation des EFE suscitent des conflits avec les élèves, voire avec leurs parents (Hutchison et Jazzar, 2007 ; Mujawamariya, 2000 et 2008), mais peu en ont fait un objet d'étude pour saisir comment les EFE reconstruisent leur savoir-faire en cette matière au travers de leurs interactions au sein de leur nouvelle écologie professionnelle.

\section{Les défis de l'évaluation des apprentissages pour les EFE au Québec}

Au Québec, l'évaluation des apprentissages soulève d'importants défis, et les enseignants doivent adapter leurs pratiques de manière continue pour soutenir le développement des compétences des élèves tout en créant les conditions de réussite du plus grand nombre (Fontaine, Savoie-Zajc et Cadieux, 2018). Or si les exigences qu'impliquent les finalités de l'évaluation des apprentissages (différenciation, évaluation formative continue, régulation des apprentissages et de l'enseignement, jugement professionnel, entre autres) sont d'importance au Québec, elles contrastent avec la culture de l'évaluation dans laquelle ont été socialisés la plupart des EFE qui s'intègrent dans les écoles québécoises. Sur ce plan, les difficultés que leur pose l'évaluation ralentiraient leur intégration harmonieuse dans les écoles. En effet, il faut savoir que les EFE ont été formés pour la plupart dans leurs pays d'origine uniquement à la fonction de sanction de l'évaluation, formule qui s'oppose nettement à la finalité d'aide à l'apprentissage valorisée au Québec (Morrissette et Diédhiou, 2017).

En d'autres mots, les EFE aborderaient leurs premières expériences d'enseignement au Québec avec un savoirévaluer, en l'occurrence un savoir-faire constitué de pratiques d'évaluation guidées par une sagesse théorique précise (Tochon, 2011), reposant sur le "paradigme de l'intuition pragmatique " (De Ketele, 1993; Perrenoud, 1991). Cela amène à considérer que l'enseignant, fort d'une expérience de terrain, en vient à se reconnaître comme le seul légitime à définir ce qui est pertinent en termes d'évaluation (ex. : critères) et même à déterminer les finalités de l'évaluation (ex. : sanctions). On l'aura compris, ce paradigme de l'évaluation, qui est celui auquel sont socialisés les EFE dans leur pays d'origine, s'oppose à celui en vigueur au Québec.

S'inscrivant dans la continuité de Morrissette et Demazière (2018), qui ont dégagé que le moteur de l'ajustement des EFE est la réaction des autres, c'est-à-dire les obstacles que ces derniers posent (parfois des conseils et des 
modélisations, mais parfois aussi des sanctions et rappels à l'ordre), cette contribution expose comment, dans les interactions de travail, les EFE ajustent leur bagage antérieur d'expériences aux finalités de l'évaluation des apprentissages valorisée au Québec, plus précisément comment ils doivent abandonner une part de ce bagage pour s'ajuster aux valorisations dans les écoles où ils s'intègrent.

\section{Une dynamique d'ajustement saisie au travers d'un principe empirique d'adaptation}

Pour analyser ce bagage d'expériences non réinvesti par les EFE sous l'effet des obstacles posés par les partenaires, je recours au concept de « définition de la situation » de Thomas (1923), c'est-à-dire la manière dont les acteurs interprètent et donnent sens aux situations qui les concernent en se coordonnant avec les définitions des autres acteurs avec qui ils interagissent. Les fondements théoriques de ce principe empirique d'ajustement se situent au cœur de la tradition sociologique de Chicago, qui postule l'indétermination des conduites humaines (Chapoulie, 2001).

Dans leurs analyses des phénomènes sociaux, les chercheurs de la tradition de Chicago ont mis en place des modèles explicatifs qui éclairent les phénomènes sociaux en s'intéressant aux manières dont les acteurs interagissent les uns avec les autres. De fait, en opposition avec la perspective fonctionnaliste qui conduirait à retenir le principe du one best way, ils conçoivent que les conduites des acteurs ne sont pas inscrites dans des schémas formels préétablis et qu'ils agissent plutôt sur la base d'une certaine souplesse, les conduites des uns et des autres étant constamment en émergence et négociées. Pour rendre compte de la confrontation dynamique entre l'expérience subjective de l'acteur et les conditions objectives dans lesquelles il est engagé, Thomas (1928) a posé un postulat, devenu célèbre : « si les hommes définissent les situations comme réelles, elles sont réelles dans leurs conséquences ${ }^{1}$ (Thomas et Thomas, p. 572). Rattaché à un univers épistémologique interprétatif, ce postulat suggère que c'est la manière dont les acteurs envisagent les situations qu'ils rencontrent qui permet d'expliquer les conduites humaines, conduites vues comme des réponses pratiques élaborées pour s'adapter aux situations compte tenu de ce qu'ils conçoivent comme étant les données significatives qui les cadrent. Par exemple, ce qu'ils anticipent des contraintes, des ressources et des réactions des autres partenaires avec qui ils interagissent infléchit leurs manières d'agir. L'interaction entre acteurs suggère ici à la fois une confrontation et une négociation pour clarifier le sens de leur objet de divergence.

Cet éclairage théorique conduit à envisager que les EFE développent des réponses adaptatives qui ne passent pas toujours par un processus réflexif afin de se tirer d'affaire lorsqu'ils rencontrent des obstacles dans leur nouveau contexte de travail. Dans l'analyse présentée ici, les obstacles sont constitués d'une partie du bagage d'expériences qui bouscule les valorisations véhiculées par les nouveaux partenaires de travail dans les activités quotidiennes. En cela, cet éclairage introduit l'idée d'une socialisation professionnelle qui n'est pas exempte des influences des partenaires de travail.

\section{Une recherche collaborative pour co-analyser la reconstruction du savoir-évaluer des EFE}

La recherche collaborative se définit comme une démarche d'investigation formelle qui sert aussi d'occasion de formation pour les participants (Desgagné, 1997 ; Morrissette, 2013). Ce modèle accorde une place importante à la négociation du contrat collaboratif et à la participation des acteurs concernés à la coconstruction du savoir. Dans le cadre de ma recherche doctorale qui visait à documenter la reconstruction du savoir-évaluer des EFE au Québec, j'ai conduit une recherche collaborative qui, tout en se greffant à une recherche sur l'intégration socioprofessionnelle d'EFE dans les écoles montréalaises (Morrissettte et Demazière, subvention du Conseil de recherches en sciences humaines [CRSH] 2015-2017), a cerné une problématique à part entière autour de leurs manières d'évaluer les apprentissages de leurs élèves. La recherche à laquelle j'ai participé à titre d'auxiliaire de recherche a impliqué 4 EFE invités à participer à un entretien biographique (Demazière, 2011) ainsi que certains de leurs partenaires de travail, introduits graduellement dans le cadre d'une série de 5 entretiens de groupe : une tutrice de stage, deux conseillers pédagogiques, une directrice d'école et un parent d'élève. Après une première analyse des verbatim issus de ces entretiens de groupe qui a permis de mieux saisir le rôle des partenaires de travail dans l'intégration des EFE, j'ai conduit une collecte de données complémentaire de manière autonome, sur

${ }^{1}$ If men define situations as real, they are real in their consequences. 
la question ciblée de l'évaluation des apprentissages, en invitant ces 4 mêmes EFE et un $5^{\mathrm{e}}$ (Békir) à participer à la démarche. Voici leur profil.

Békir : originaire du Maroc où il a enseigné pendant 15 ans, cet enseignant de mathématiques en était à sa quatrième année d'enseignement au Québec, avec un contrat à $100 \%$.

Fatima : originaire de Tunisie où elle a enseigné pendant 16 ans, cette enseignante de sciences naturelles en était à sa troisième année d'enseignement au Québec, avec un contrat à $75 \%$.

Nabila : originaire d'Algérie où elle a enseigné pendant 2 ans, cette enseignante de français en était à sa troisième année d'enseignement au Québec, avec un contrat à $100 \%$.

Sadia: originaire de l'Algérie où elle a enseigné pendant 13 ans, cette enseignante de mathématiques en était à sa quatrième année d'enseignement au Québec, avec un contrat à $100 \%$.

Vika : originaire de Moldavie où elle a enseigné pendant 3 ans, cette enseignante d'anglais en était à sa quatrième année d’enseignement au Québec, avec un contrat à $100 \%$.

Ils ont d'abord été invités à un entretien individuel de type récit de pratique (Desgagné, 2007) au cours de l’hiver 2016, dans le cadre duquel ils ont été incités à décrire les transformations de leurs façons de conduire l'évaluation à la suite de leur intégration à l'école montréalaise. Ils ont ensuite été invités à participer à trois entretiens de coanalyse en groupe, centrés sur des épisodes exemplaires des tensions vécues lorsqu'ils ont initialement reconduit leur bagage antérieur d'expériences en matière d'évaluation des apprentissages dans le contexte des écoles montréalaises. Cette contribution repose donc sur le matériau constitué par ces 9 entretiens individuels et ces 8 entretiens de groupe en lien avec ma recherche doctorale (Diédhiou, 2018). La stratégie analytique a consisté en une démarche de théorisation par induction analytique (Becker, 2000 ; Blais et Martineau, 2006 ; Glaser et Strauss, 2017), soit une production d'énoncés théoriques empiriquement fondés à partir des données. En effet, en raison du projet analytique qui visait à documenter le bagage d'expériences que les EFE ne réinvestissent pas durant leur processus d'intégration socioprofessionnelle, la méthode du raisonnement par cas de Becker (2016) a été mobilisée. Ainsi, la comparaison continue de l'expérience au Québec avec celle du pays d'origine a permis de faire émerger divers usages de l'évaluation contestés par les partenaires de travail. Je présente ici la manière dont ces interactions au travail ont façonné le bagage d’expériences que les EFE ne réinvestissent pas dans les écoles montréalaises.

\section{Des résultats sur l'abandon d'usages de l'évaluation contestés par les partenaires de travail}

Les résultats éclairent un processus adaptatif important dans la reconstruction du bagage d'expériences des EFE : l'abandon d'un savoir-évaluer non opératoire, à savoir des usages de l'évaluation contestés par les élèves, les parents ou les directions d'école. Ces usages contestés concernent la mobilisation de l'évaluation comme moyen de pression sur les élèves, d'une part, et comme outil au service du " tri social ", d'autre part. Pour chacun d'eux, je présente les processus adaptatifs qui émergent du travail d'interprétation que les EFE ont fait des situations en interaction avec leurs partenaires de travail. Comme on le verra, ce travail de définition des situations rencontrées les a conduit à abandonner certains usages de l'évaluation considérés comme pertinents dans leurs pays d'origine au profit d'un alignement sur ce qui est valorisé dans leur nouvelle écologie professionnelle d'appartenance.

\section{La mobilisation de l'évaluation comme moyen de pression sur les élèves}

Un premier usage de l'évaluation des apprentissages par les EFE contesté par les partenaires de travail au Québec concerne la mobilisation de l'évaluation en tant que violence symbolique légitime, c'est-à-dire qu'elle constitue un instrument de pouvoir au service de leur domination sur leurs élèves. L'évaluation instituerait l'autorité de l'enseignant, c'est-à-dire une forme de respect imposé et à laquelle les élèves devraient se soumettre. Dans les écoles montréalaises, les EFE ont reconduit cette domination en laissant planer un climat de peur, par la menace de l'évaluation surprise susceptible de se faire à tout moment en classe. Cette façon de faire a vite été décriée par leurs nouveaux partenaires de travail, en priorité par les élèves qui ont vivement protesté, révélant le caractère non opératoire de cette pratique en contexte montréalais. Par exemple, les élèves ont contesté les manières 
d'évaluer qu'ils considéraient comme une menace à leur bien-être ou une atteinte à leur estime de soi. Les lignes qui suivent présentent les deux processus qui ont joué dans l'abandon de l'usage de l'évaluation comme moyen de pression sur les élèves: la confrontation au renversement de la charge de la pression et la socialisation aux interdits et aux valorisations sociales partagées.

\section{Un abandon par confrontation au renversement de la charge de la pression}

Comme l'ont rapporté les EFE, dans leurs pays d'origine, ils mobilisaient parfois l'évaluation dans une logique de régulation des conduites des élèves et de maintien de la discipline dans leur classe. En expliquant leurs premières expériences d'enseignement au Québec, plusieurs EFE sont revenus sur leurs tentatives d'utiliser la pression par l'évaluation pour obtenir des élèves l'engagement souhaité dans les activités d'apprentissage. En réponse, ils ont enregistré chez leurs élèves une forme d'indolence et un refus de collaboration qui rompaient avec ce qu'ils avaient connu jusque-là. Ces réactions des élèves, pour lesquelles ils n'étaient pas outillés, ont fait peser la peur sur eux. Ils ont fait face à un renversement de la charge de la pression, craignant que le refus d'obéir manifesté par leurs élèves les expose aux jugements des membres de leur écologie professionnelle, qui les verraient comme " incompétents » (Békir). C'est ce que relève Nabila, en expliquant la situation délicate dans laquelle elle se retrouve en découvrant qu'elle ne peut pas utiliser l'évaluation comme moyen d'intimider ses élèves, alors qu'elle a l'obligation professionnelle de les faire travailler : « ce n'est pas évident; ne pas sanctionner quand tes élèves ne coopèrent pas? tu es comme sans moyen » (Nabila).

Ainsi, ils ont fait face au défi d'infléchir les conduites des élèves dans le sens d'une participation plus active à leurs apprentissages sans pour autant recourir au pouvoir de sanction permettant d'utiliser l'évaluation pour discipliner les élèves. Lorsque certains enseignants ont essayé d'y recourir, non pas pour sanctionner mais pour avoir plus d'attention de la part de leurs élèves, ils ont vite constaté l'échec de leur stratégie. La pression par l'évaluation ne semble avoir aucun effet sur eux, comme en témoigne Vika : « mes élèves de secondaire 4, j'ai essayé de leur parler de l'examen ministériel pour leur mettre un peu de pression et les pousser à se concentrer dans l'activité, ça n'a rien donné ; [...] ici, les élèves n'ont pas peur de l'évaluation ». Anticipant des conséquences néfastes sur leur carrière issues des protestations et des refus de collaborer des élèves, les EFE se seraient vus contraints d'abandonner cette manière d'utiliser l'évaluation pour obtenir les conduites souhaitées. Face à la pression des élèves, ils ont choisi de s'aligner sur les valorisations partagées, entre autres en négociant avec les élèves leur participation aux activités d'enseignement et d'apprentissage.

\section{Un abandon par socialisation aux interdits et aux valorisations sociales partagées}

En faisant une analyse comparative des contextes d'enseignement dans lesquels ils ont travaillé, les EFE notent que les attentes qu'ils avaient quant à l'obéissance à leur autorité ne semblent pas fonctionner dans les relations avec leurs élèves montréalais en raison des valorisations sociales divergentes. En fait, ce qui semble partagé dans leur nouvelle écologie professionnelle, par exemple des rapports plus symétriques entre enseignant et élèves et l'importance du bien-être de leur enfant, fait nettement obstacle à toutes les formes de violence symbolique à l'endroit des élèves, l'évaluation ne pouvant être au service de la sanction de la conduite des élèves. C'est le tableau que présente Nabila, en mettant en parallèle les deux contextes de travail qu'elle a connus : " chez nous, [...] la menace de l'évaluation [...] fait que les élèves accordent le respect même s'ils sont plus forts que l'enseignant [...] ; ici [...] tu n'es pas l'autorité toute puissante ; l'évaluation, tu ne peux pas menacer avec ça; ce n'est pas une arme que tu vas brandir pour avoir ce que tu veux de tes élèves " (Nabila).

Cette comparaison ouvre un pan sur une reconnaissance de l'autorité qui ne va pas dans le sens de positions plutôt asymétriques, comme ce que les EFE ont connu dans leur pays d'origine. Elle suggère que dans le contexte des relations enseignant-élèves dans les écoles montréalaises, l'autorité n'est pas reconnue a priori par le pouvoir d'imposer l'obéissance, ni par l'intimidation : " ici, tu ne peux pas utiliser l'évaluation pour intimider tes élèves ; [...] c'est interdit; [...] dans la formation en évaluation, on nous dit l'importance d'être en cohérence avec la politique d'évaluation; elle présente ce qu'on attend dans l'évaluation des apprentissages » (Fatima). 
Cet extrait montre que la connaissance des finalités de l'évaluation (aide à l'apprentissage, reconnaissance des compétences) par tous les partenaires scolaires est une barrière importante pour des pratiques d'évaluation autres que celles socialement partagées. C'est ce dont témoigne Nabila dans la suite de son propos: "I'évaluation, ça te sert pour autre chose, aider les élèves " (Nabila). Cette autre perspective de l'évaluation conduirait les EFE à envisager la négociation comme processus important dans la conduite des activités d'enseignementapprentissage : « [...] ici, je négocie tout avec les élèves : les activités, l'évaluation, etc. ; [...] c'est ça que j’ai trouvé génial, parce qu'on considère l'élève comme une personne respectable » (Sadia).

\section{La mobilisation de l'évaluation comme outil au service du « tri social » des élèves}

Un autre usage de l'évaluation des apprentissages par les EFE, contesté par les partenaires de travail au Québec, concerne sa mobilisation comme outil de "tri sélectif » pour classer les élèves les uns par rapport aux autres. Suivant cette même finalité de sélection sociale, l'évaluation servirait à "écrémer ", c'est-à-dire à écarter plus rapidement les élèves les moins performants du système scolaire, tel que le formule Fatima : « [...] plus on avance, plus les effectifs diminuent; on forme vraiment l'élite". Aussi, pour "contraindre " les élèves à travailler davantage, c'est-à-dire les empêcher de "faire dans la paresse » (Békir), les enseignants utiliseraient cette façon de faire l'évaluation. Les lignes qui suivent présentent les deux processus qui ont joué dans l'abandon de l'usage de l'évaluation comme outil au service du " tri social » : la prévision de la reddition de comptes aux parents et la prise en compte du respect de l'estime de soi des élèves.

\section{Un abandon par prévision de la reddition de comptes aux parents}

Une des façons de faire l'évaluation rapportée par les EFE consiste en une sur-dépréciation des performances des élèves, c'est-à-dire ne pas leur donner des notes très bonnes « [...] pour les maintenir dans l'idée qu'ils ne sont pas assez bons et afin qu'ils travaillent plus fort » (Békir). Cette vision partagée par le groupe d'EFE façonnait le savoirévaluer de plusieurs d'entre eux, qui trouvaient que c'était la meilleure façon de préparer leurs élèves à un " monde de compétition qui ne fait de la place qu'aux seuls meilleurs " (Nabila). Considérant leur rôle d'évaluateur comme étant de "familiariser les élèves avec un système d'évaluation qui ne leur fera pas de cadeau » (Fatima), les EFE en provenance du Maghreb en particulier légitimaient leurs façons de faire l'évaluation en convoquant une convention partagée dans le pays d'origine, c'est-à-dire " qui aime bien, châtie bien ». Or cette formule (et les pratiques qui en relèvent) ne semble pas opératoire dans leur nouveau milieu de travail, qui exige que l'enseignant justifie ses évaluations auprès de ses partenaires scolaires. La reddition de comptes aux parents concernant les résultats de l'évaluation des apprentissages des élèves semble un moment redouté dans les écoles. Elle impliquerait pour les EFE, comme pour tous leurs collègues, de prendre certaines précautions en amont pour éviter des conflits potentiellement préjudiciables à leur carrière.

C'est à cette convention importante que Vika est socialisée au travers de la mésaventure de sa compatriote Linda, une immigrante qui enseigne l'espagnol dans une école montréalaise. Cette enseignante y a vu ses manières d'évaluer fortement remises en cause au cours de ses premières années. Pensant bien faire en ne donnant pas de notes élevées et en s'arrangeant pour que les performances des élèves puissent permettre de les classer selon quatre groupes - les excellents (une très faible minorité), les bons (un groupe un peu plus nombreux que le premier), les moyens (la majorité) et les faibles (environ le double du premier groupe) -, elle a présenté ses notes à sa directrice. Celle-ci lui aurait fait vite comprendre "qu'elle ne peut pas les accepter [les notes]; c'est trop catastrophique ; les parents vont se plaindre » (Vika).

Cette réaction de la directrice l'aurait alertée sur la réception des manières d'évaluer dans son nouveau milieu de travail : les évaluations qui se démarquent par un fort taux d'échec ne passent pas. Ce rejet étonne les EFE, dont l'expérience avec l'évaluation est toute autre dans leur pays d'origine: les parents ne se plaignaient jamais des mauvaises notes attribuées par les enseignants. Ce "faux-pas " commis par sa compatriote lui aurait servi de référence pour prendre la mesure des tensions au cœur de l'évaluation des apprentissages. "[...] elle m'a dit “'j'étais choquée parce que je ne savais plus quoi faire avec ces notes; est-ce que je dois les modifier pour plaire à la directrice; pour moi ce n'est pas éthique; je n'avais pas inventé ces notes; c'est le résultat du travail des élèves" » (Vika). 
Cet extrait reflète bien la tension entre des visions divergentes de l'évaluation, tension qui interpelle l'éthique professionnelle. Pour l'EFE, les notes sont le produit du travail des élèves. Donc, même si elles sont faibles (basses), l'enseignant n'a pas le droit de les modifier. C'est d'ailleurs de cette manière qu'il fournirait un gage de son intégrité professionnelle. Cette vision ne semble pas partagée par la direction, qui évaluerait la situation dans le sens de la reddition de comptes aux parents. II s'agit là d'une distribution différente du pouvoir, qui présente les parents comme les gardiens du respect des valorisations en lien avec la réussite des élèves. Le contraste est bien net entre la vision de l'évaluation portée par les EFE - celle de " compétition » et de surpassement des élèves les uns par rapport aux autres - et l'accueil que lui réservent les directions d'école, qui y voient une "forme de sanction des élèves par les notes " (Vika). Pour échapper aux plaintes des parents et face aux élèves qui revendiquent de meilleures notes, les EFE finissent par s'aligner, comme l'exprime Nabila : « maintenant, je donne des $100 \%,[. .$.$] même s'il y a des fautes »$.

\section{Un abandon par prise en compte du respect de l'estime de soi des élèves}

Dans une mise en perspective temporelle favorisée par les comparaisons établies en situation d'entretien, les EFE ont revisité certaines de leurs pratiques professionnelles significatives de leurs représentations de l'évaluation dans leur pays d'origine mais peu opératoires au Québec. Ils ont trouvé que leur reproduction n'était plus possible puisqu'elles reflétaient pour les partenaires scolaires une humiliation des élèves. Par exemple, ils ont abandonné leur manière de rendre les résultats des tests utilisée dans leur pays d'origine, en faisant le constat des conséquences associées à leur reconduction.

Nous on donnait les notes par ordre : d'abord les meilleurs élèves, ceux qui ont bien travaillé, on les félicite ; on les valorise ; on finit avec les plus faibles notes; on les critique pour qu'ils travaillent mieux la prochaine fois ; [...] ici c'est quelque chose que je ne peux pas faire; tu risques d'être convoquée à la police parce que c'est une faute grave; [...] il y a des parents qui vont porter plainte ; [...] dans mon pays, dire les notes à haute voix, classer les élèves, c'est normal (Vika).

On voit bien ici le théorème de Thomas, car dans la réalité, la police ne se mêlerait pas de ce problème. Mais parce que les EFE le croient, ils agissent pour l'éviter. Cet extrait suggère ainsi que, devant la crainte des sanctions, les EFE s'ajustent dans le sens de l'alignement souhaité, renonçant aux pratiques évaluatives qui fonctionnaient dans leur pays d'origine. Il faut remarquer que les valorisations socialement partagées se présentent comme des obstacles à la reconduction de pratiques d'évaluation pourtant considérées dans les pays d'origine des EFE comme pertinentes pour inciter les élèves à donner le meilleur d'eux-mêmes. Les manifestions de ces mêmes pratiques sont prohibées au Québec : «tu ne peux pas faire de classement par ordre de mérite ici ; ce n'est pas accepté [...] la direction te dira que ça va créer des plaintes de parents ; c'est pas bon pour ton intégration » (Sadia). Considérée comme une atteinte à la personnalité de l'élève, donc une infraction lourde de conséquence sur sa motivation, les parents en défendent l'application. Le respect de l'estime de soi de l'enfant semble une valorisation non négociable pour les parents. Considérant le fait d'avoir des problèmes avec les parents comme une manière de compromettre leurs chances de faire l'expérience d'une intégration harmonieuse dans les écoles, plusieurs renoncent à mobiliser l'évaluation dans le sens de la sélection sociale. C'est ce dont témoigne Nabila qui relève :

ici, tu ne peux même pas utiliser le travail d'un élève pour donner un exemple aux autres : c'est comme si tu le valorises sur les autres ; [...] ça c'est interdit ; [...] donc pour avoir la paix avec les parents, tu dois éviter toute pratique qui mettrait les élèves en « compétition » les uns avec les autres. (Nabila)

Cet extrait reflète que l'estime de soi de l'élève au Québec est une valorisation incontournable. Les objections des partenaires scolaires ici présentés reflètent que les pratiques d'évaluation qui affectent négativement la personnalité des élèves, voire leur bien-être, ne sont pas tolérées. Pour les parents, il semblerait surtout que c'est par souci de préserver les intérêts de leur enfant, dont ils n'aimeraient pas voir les efforts anéantis par une comparaison avec les autres élèves. Les EFE expliquent que les parents refuseraient aussi tout jugement de valeur négatif accompagnant la note de l'élève. «Ici, tu ne peux pas dire d'un enfant pas travailleur, qui a des notes faibles, que c'est un paresseux ; [...] chez nous tu peux le faire sans rien craindre ; ici tu vas avoir une plainte qui va tomber parce que c'est humiliant pour l'enfant ; tu ne respectes pas sa personnalité » (Békir). 
Ces propos suggèrent que les interprétations que les partenaires de travail font de certaines façons d'utiliser l'évaluation représentent des indices auxquels les EFE se réfèrent pour s'ajuster. Parfois, leurs expériences personnelles en tant que parents les conduisent à comprendre que l'évaluation appliquée dans le sens de la sélection sociale anéantit l'image et l'estime de soi de leur enfant. C'est ce qu'évoque Fatima, en rapportant l'expérience d'un parent d'élève qui leur a partagé son vécu, ses propos l'ayant sensibilisée aux effets de l'évaluation et des rétroactions qui l'accompagnent sur la motivation des élèves.

Dans mon pays je classais les copies, les forts d'abord et les faibles ensuite; [...] l'expérience de Lucie [prénom fictif d'un parent d'élève] me fait beaucoup réfléchir; [...] c'est [...] déstabilisant pour un parent de voir sa fille en larmes, complétement anéantie; je me mets à sa place comme maman; [...] ça m’a [...] convaincue que cette pratique était à abandonner. (Fatima)

On constate que sur le coup, avec les sanctions, les EFE s'ajustent mais n'adhèrent pas aux nouvelles valorisations telles qu'elles apparaissent dans les réactions des personnes avec qui ils interagissent. Cependant, avec une explication, un point de vue autre qui rejoint leur expérience de parent, ils finissent par donner du sens à ce qui ne se fait pas en contexte montréalais et peuvent dès lors adhérer à cette nouvelle norme. Le vécu des parents, lorsqu'il est explicité, contribuerait ainsi à socialiser les EFE aux conséquences de l'évaluation sur la motivation des élèves.

\section{Discussion conclusive : une faible marge de manœuvre dans la pratique de l'évaluation}

Prolongeant les recherches qui montrent que pour survivre aux obstacles qu'ils rencontrent, les EFE bricolent des stratégies adaptatives, en particulier le bénévolat dans les écoles ou la sollicitation de l'accompagnement d'un mentor informel (Duchesne, 2008; Mujawamariya, 2000 et 2008; Peeler et Jane, 2005), cette contribution éclaire un processus adaptatif important, à savoir l'abandon d'un savoir-évaluer non opératoire, qui se décline, comme on l'a vu, dans différentes circonstances. Elle fait ressortir que le bagage d'expériences non réinvesti par les EFE peut être éclairé à partir du sens qu'ils coconstruisent dans leurs rapports avec leurs différents partenaires scolaires. L'intensité et la récurrence des contestations et critiques auxquelles ils ont à faire face les auraient amenés à ne plus mobiliser des manières d'évaluer contestées, et ce, même si plusieurs d'entre eux demeurent convaincus de leur légitimité pour obliger les élèves à apprendre et performer. En d'autres mots, ils s'alignent sur le fait que l'estime de soi de l'élève au Québec est une valorisation incontournable, mais ils ne changent pas leurs manières de considérer l'évaluation.

Ma recherche montre que l'idée du peu de marge de manœuvre en matière d'évaluation des apprentissages est liée au fait qu'au Québec, la norme par excellence est le bien-être de l'enfant et que l'évaluation est vue comme la menaçant de manière importante. On la vu, la préservation de cette norme vient avec une conception de l'évaluation et un nouveau rapport de places (Kerbrat-Orrecchioni, 1990), c'est-à-dire une interdépendance qui demande pour les EFE d'abandonner pour pouvoir apprendre. II faut noter que l'imbrication des prescriptions avec les valorisations sociales réduit la marge de manœuvre des EFE, qui se considéraient jusque-là comme les seuls légitimes à parler d'évaluation. Ils doivent composer avec des parents et des élèves, c'est-à-dire des " profanes » qui essaient d'avoir le contrôle sur la " juridiction " (Abbot, 1988 et 2003) de l'évaluation par la sanction des pratiques des EFE, qu'ils jugent " transgressives ». L'abandon du savoir-évaluer relevant de l'expérience antérieure pour s'aligner sur les valorisations socialement partagées dans leur nouveau contexte de travail peut dès lors paraître délégitimant pour plusieurs : ils sont obligés de choisir cette alternative, la seul viable pour obtenir la reconnaissance de leurs partenaires de travail et pour devenir membre d'une nouvelle communauté professionnelle.

Finalement, le rapport de places qui relève de cette réduction de la marge de manœuvre des EFE concernant l'évaluation conduit à interpréter leur apprentissage en contexte d'intégration socioprofessionnelle comme une mise à l'épreuve de leur bagage d'expériences. Cela explique que les processus adaptatifs en lien avec l'abandon des manières d'évaluer contestées sont construits hic et nunc par les EFE, à partir de la trame des situations qu'ils rencontrent et au croisement des interprétations négociées (Morrissette, 2009) avec les différents partenaires de travail. 


\section{Références bibliographiques}

Abbott, A. D. (2003). Écologies liées : à propos du système des professions. Dans P.-M. Menger (dir.), Les professions et leurs sociologies : modèles théoriques, catégorisations, évolutions : actes du Colloque de la Société française de sociologie, Paris, octobre 1999 (p. 29-53). Paris : Maison des sciences de l'homme.

Abbott, A. (1988). The System of Professions : an Essay on the Division of Expert Labor. Chicago : University of Chicago Press.

Adméé - Canada (2014). L'évaluation au cœur de tensions. 36 e session d'études de l'Adméé-Canada, les 13 et 14 novembre 2014, à Montréal.

Becker, H. S. (2016). La bonne focale. De l'utilité des cas particuliers en sciences sociales. Paris : La Découverte.

Becker, H.S. (2000). L'enquête de terrain : quelques ficelles du métier. Sociétés contemporaines, 40, $151-164$.

Blais, M. et Martineau, S. (2006). L'analyse inductive générale : description d'une démarche visant à donner un sens à des données brutes. Recherches qualitatives, 26(2), 1-18.

Caza, P.E. (2019). Une solution à la pénurie d’enseignantes [En ligne]. Actualités UQÀM, 4 pages https://www.actualites.uqam.ca/2019/une-solution-a-la-penurieenseignantes?utm_campaign=UQAMHEBDOetutm_medium=emailetutm_source=27MARS2019

Chapoulie, J.M. (2001). La tradition sociologique de Chicago. 1892-1961. Paris : Seuil.

Charara, Y. et Morrissette, J. (2018). Les dispositifs d'intégration socioprofessionnelle des enseignants immigrants au Québec: un état de la question éclairé par la littérature internationale. Dans A. Manço et J. Gatugu (dir.), Insertion des travailleurs migrants. Efficacité des dispositifs (p. 183-198). Paris : L'Harmattan.

Cloutier, P. (2018). Une pénurie d'enseignants qui s'aggrave [En ligne]. Le Soleil, 12 mars. https://www.lesoleil.com/actualite/education/une-penurie-denseignants-qui-saggravec966ce80ff7008a644523e2f1b631b3f

De Ketele, J. M. (1993). L'évaluation conjuguée en paradigmes. Revue française de pédagogie, 103, 59-80.

Demazière, D. (2011). L'entretien biographique et la saisie des interactions avec autrui. Recherches qualitatives, $30(1), 61-83$

Desgagné, S. (1997). Le concept de recherche collaborative : l'idée d'un rapprochement entre chercheurs universitaires et praticiens enseignants. Revue des sciences de l'éducation, 23(2), 371-393.

Desgagné, S. (2007). Le défi de coproduction de savoir en recherche collaborative. Autour d’une démarche de reconstruction et d'analyse de récits de pratique enseignante. Dans M. Anadon (dir.) La recherche participative. Multiples regards (p. 89-121). Québec: Presses de l’Université du Québec.

Deters, P. (2006). Immigrant teachers in Canada: Learning the language and culture of a new professional community. Actes du $5^{e}$ Colloque de l'AELFE, 14 au 16 septembre 2006. Saragosse, Espagne : AELFE. http://citeseerx.ist.psu.edu/viewdoc/download?doi=10.1.1.464.4686\&rep=rep1\&type=pdf

Duchesne, C. (2008). Difficultés d'insertion professionnelle en enseignement : le rôle des conceptions d'étudiants étrangers inscrits dans un programme de formation des maîtres. Formation et pratiques d'enseignement en questions, 8, 119-139.

Fontaine, S., Savoie-Zajc, L. et Cadieux, A. (2018). Évaluer les apprentissages. Démarche et outils d'évaluation pour le primaire et le secondaire. Québec : Les éditions CEC.

Glaser, B. G., et Strauss, A. L. (2017). La découverte de la théorie ancrée. Stratégies pour la recherche qualitative. Paris : Armand Colin.

Goodson, I., Thiessen, D. et Bascia, N. (1997). Making a difference about difference: The lives and careers of racial minority immigrant teachers. Canadian Journal of Education, 22(4), 462-465. 
Gouvernement du Québec (2018). Politique québécoise en matière d'immigration, de participation et d'inclusion. Ensemble nous sommes le Québec.

http://www.midi.gouv.qc.ca/publications/fr/dossiers/RecueilStatistiques.pdf

Hutchison, C.B., et Jazzar, M. (2007). Selecting, training, and evaluating teacher mentors for international teachers in American schools. Phi Delta Kappan, 88(5), 368-373.

Immigration, Réfugiés et Citoyenneté Canada. (2018). Rapport annuel au Parlement sur l'immigration. Ottawa: Gouvernement du Canada. https://www.canada.ca/content/dam/ircc/migration/ircc/francais/pdf/pub/rapport-annuel-2018.pdf

Jabouin, S., et Duchesne, C. (2012). Le difficile parcours d'insertion des enseignants issus de l'immigration dans la profession enseignante en Ontario. Revue d'éducation de l'Université d'Ottawa, 2(1), 4-5.

Kerbrat-Orrecchioni, C. (1990). Les interactions verbales. Paris : Armand Colin.

Martineau S., et Vallerand, A.-M. (2007). Chronique sur l'insertion professionnelle en enseignement: la question des enseignants de migration récente. Formation et profession, 13(2), 51-54.

Ministère de l'éducation et de l'enseignement supérieur (MEES) (2015). Conditions et modalités pour obtenir un permis d'enseigner au Québec. Pour les titulaires d'une autorisation d'enseigner obtenue à l'extérieur du Canada. Québec: Gouvernement du Québec http://www.education.gouv.qc.ca/fileadmin/site_web/documents/reseau/formation_titularisation/Conditions_permis_enseigner_ExtCa-nada_document_info_FR.pdf

Morissette, J. (2013). Recherche-action et recherche collaborative : Quel rapport aux savoirs et à la production de savoirs? Recherches participatives, 25(2), 35-49.

Morrissette, J. (2009). La portée d'une perspective socioculturelle de l'évaluation formative: vers l'élargissement d'une conceptualisation. Mesure et Évaluation en Éducation, 32(2), 1-23.

Morrissette, J. et Demazière, J. (2018). Dualité des processus de socialisation professionnelle des enseignants formés hors Québec. Entre imposition et appropriation. Alterstice, 8(1), 95-106.

Morrissette, J., Demazière, D., Diédhiou, S .B. M. et Sèguéda, S. (2018). Les expériences d'intégration professionnelle d'enseignants migrants à Montréal. Rapport de recherche soutenu par le Conseil de recherche en sciences humaines du Canada et la Commission scolaire Marguerite-Bourgeois, Montréal. https://cipcd.ca/wp-content/uploads/2014/04/Rapport-de-recherche-Morrissette-Demazi\%C3\%A8reintergration-professionnelle-ens-migrants.pdf

Morrissette, J. et Diédhiou, S. B. M. (2017). Le caractère collectif de la (re)construction du savoir-évaluer des enseignants migrants qui s'intègrent dans les écoles québécoises. Mesure et évaluation en éducation, 40(2), 33-56.

Morrissette, J., Diédhiou, S. B. M. et Charara, Y. (2014). Un portrait de la recherche sur l'intégration socioprofessionnelle des enseignants formés à l'étranger. Rapport de recherche déposé au Centre d'intervention pédagogique en contexte de diversité de la Commission scolaire Marguerite-Bourgeois, Montréal. www.cipcd.ca/wp-content/uploads/2014/10/Rapport-Morrissette-et-al-VF-20-oct-2014.pdf

Mujawamariya, D. (2008). Les difficultés d'insertion professionnelle des enseignants de sciences de race noire en milieu francophone ontarien: un pattern singulier ? Dans L. Portelance, J. Mukamurera, S. Martineau et C. Gervais (dir.), L'insertion dans le milieu scolaire, une phase cruciale du développement professionnel de l'enseignant (p. 145-161). Québec : Presses de l'Université Laval.

Mujawamariya, D. (2000). Ils ne peuvent pas enseigner dans nos écoles : le dilemme des étudiants-maîtres des minorités visibles nés au Canada. Reflets : revue d'intervention sociale et communautaire, 6(2), 138-165.

Myles, J., Cheng, L., et Wang, H. (2006). Teaching in elementary school: Perception of foreign-trained teacher candidates of their teaching practicum. Teaching and Teacher Education, 22(2), 233-245. 
Niyubahwe, A. (2015). L'expérience d'insertion professionnelle des enseignantes et enseignants de migration récente au Québec (Thèse de doctorat inédite). Université de Sherbrooke, Sherbrooke.

Niyubahwe, A., Mukamurera, J., et Jutras, F. (2014). L'expérience de transition professionnelle des enseignants de migration récente au Québec. Revue canadienne de l'éducation, 37(4), 1-32.

Niyubahwe, A., Mukamurera, J., et Jutras, F. (2013). Professional Integration of Immigrant teachers in the school system: A literature review. Revue des sciences de l'éducation de McGill, 48(2), 279-296.

Perrenoud, P. (1991). Pour une approche pragmatique de l'évaluation formative. Mesure et évaluation en éducation, 4(13), 49-81.

Phillion, J. (2003). Obstacles to accessing the teaching profession for immigrant women. Multicultural Education, 11(1), 41-46.

Thomas, W.I. (1923). The unadjusted girl : with cases and standpoint for behavior analysis. Boston, MA. : Little, Brown, and Co.

Thomas, W. I. et Thomas, D. D. S. (1928). The child in america ; Behavior problems and programs. New York : Knopf.

Tochon, F. V. (2011). Le savoir-évaluer comme politique éducative : Vers une évaluation plus profonde. Mesure et évaluation en éducation, 34(3), 137-160.

Services Canada (2015). Enseignants aux niveaux primaire et préscolaire. Emploi-Avenir Québec [En ligne] http://www.servicecanada.gc.ca/fra/qc/emploi_avenir/statistiques/4142.shtml\#perspectives

Wang, T. (2003). Cultural dissonance and adaptation: a study of Chinese immigrant teachers coping with cultural differences in Toronto schools. Ontario : University of Toronto, Ontario Institute for Studies in Education.

Zietsma, D. (2010). Immigrants exerçant des professions réglementées. Perspective, Février ( ${ }^{\circ}$ 75-001-X). Ottawa : Statistique Canada. 